understanding seroprevalence within an occupational cohort by detecting past immune response.

Objectives We conducted serological SARS-CoV-2 antibody testing from October-November 2020 to estimate the SARS$\mathrm{CoV}-2$ seroprevalence among firefighters/paramedics in Orange County.

Methods OC firefighters employed at the time of the surveillance activity were invited to participate in a voluntary survey that collected demographic, occupational, and previous COVID-19 testing data, and a SARS-CoV-2 immunoglobulin (Ig)G antibody blood test. We collected venous blood samples using mobile phlebotomy teams that traveled to individual fire stations, in coordination with an annual tuberculosis testing campaign for firefighters employed by OC Fire Authority (OCFA), and independently for firefighters employed by cities. We estimated seroprevalence and assessed several potential predictors of seropositivity.

Results The seroprevalence was 5.3\% among 923 OCFA personnel tested, with $92.2 \%$ participating. Among firefighters self-reporting a previous positive COVID-19 test result, twenty-one $(37 \%)$ were no longer positive. There were no statistically significant differences in demographic characteristics between cases and non-cases. Work city was a significant predictor of case status $(p=0.015)$. Seroprevalence $(4.8 \%)$ was similar when aggregated across seven city fire departments (42-65\% participation). In total, 1,486 OC fire personnel were tested.

Conclusion Using a strong serosurvey design and large firefighter cohort, we observed a SARS-CoV-2 IgG seroprevalence of $5.3 \%$. The seroprevalence among OC firefighters in October 2020 was lower than the general county population estimated seroprevalence (11.5\%) in August. The difference may be due in part to safety measures taken by OC fire departments at the start of the pandemic, as well as differences in antibody test methods and/or duration of antibody response.

\section{0-481 COVID-19 INFECTION AMONG HEALTHCARE WORKERS AT MALAYSIA HOSPITALS}

'Abdul Aziz Harith, Mohd Hafiz Ab Gani, Rob Griffiths, Nor Aishah Abu Bakar, Azlihanis Abdul Hadi, Maznieda Mahjom. ${ }^{1}$ University of Otago Wellington, New Zealand

\subsection{6/OEM-2021-EPI.37}

Introduction Healthcare workers are at a substantially increased risk of being infected by COVID-19 patients. However, risk of being infected is depending on the critical phase of the pandemic, patients with COVID-19 might not be the absolute source of infection. Health workers could also be exposed to infected colleagues, infected family members, lives in communities of active transmission, or infected contacts during crowded events such as wedding reception and religious gathering.

Objectives To explore the epidemiology data of COVID-19 infection among health care workers at Malaysia Hospitals especially on patterns of transmission and characteristics.

Methods A cross-sectional surveillance study among infected COVID-19 healthcare workers working at Malaysia government hospitals.

Results 1608 healthcare staffs at hospitals have been notified with COVID-19 infection in year 2020. By proportion, nursing occupation contribute up to $40.5 \%$, followed by medical doctor (20.8\%), healthcare assistant (9.7\%), medical doctor assistant (9.1\%), medical specialist (3.2\%) and hospital administrative assistant $(2.8 \%)$. Most of cases were reported from Sabah (39.8\%), Selangor (27.5\%), Wilayah Persekutuan Kuala Lumpur \& Putrajaya (6.7\%), Sarawak (6.0\%), Perak (5.6\%) and Johor (4.7\%). By gender, seven out of ten infected healthcare staffs were female and majority of them have no comorbidity (87\%). In addition, rate of COVID-19 infection among healthcare workers was proportionately increased with rate of COVID-19 infection among community. Investigation by health authorities found 43.2\% of COVID-19 infection cause by community, $36.3 \%$ occurred between staff to staff and $17 \%$ occurred between patients to staff.

Conclusion Preponderance of infection has occurred within hospital environment. Occupational Safety and Health Unit should set up a good engagement with healthcare staff and effective strategies to protect and support the health, safety and wellbeing of staff through deep-rooted assessment of standard practice procedure especially in nursing and care activities. Digital contact tracing could improve contact tracing within hospital setting.

\section{Disease Surveillance}

\section{0-24 LUNG AND BLADDER CANCER SURVEILLANCE AMONG CONSTRUCTION WORKERS IN DIESEL ENGINE EXHAUST EXPOSED OCCUPATIONS IN ONTARIO, CANADA}

'Stephanie Ziembicki, Victoria H Arrandale, Nathan DeBono, Mamadou Dakouo, Tracy Kirkham, Paul Demers. 'Ontario Health, Canada

\subsection{6/OEM-2021-EPI.38}

Introduction Diesel engine exhaust (DEE) is a lung and bladder carcinogen and one of the most common carcinogenic exposures in Canada with over 900,000 Canadians exposed at work, according to CAREX Canada. Construction workers are an understudied group despite suspected high DEE exposure; most research on DEE has been conducted in transportation and mining industries.

Objectives This study estimates incidence rates for lung and bladder cancer in construction occupations with probable DEE exposure using the Occupational Disease Surveillance System (ODSS).

Methods The ODSS includes $\sim 2.2$ million Ontario workers identified through workers' compensation claims (1983-2014). Workers were followed for cancer diagnoses through linkage with the Ontario Cancer Registry (1964-2016). DEE-exposed construction occupations were identified using Canadian Classification Dictionary of Occupation code descriptions. Coxproportional hazards models were used to estimate hazard ratios (HR) and 95\% confidence intervals (CI), adjusted for age, birth year, and sex.

Results We identified 3980 lung and 1566 bladder cases among construction trades occupations. Compared to all other ODSS workers, construction trades occupations had small elevations in lung $(\mathrm{HR}=1.08,95 \% \mathrm{CI} 1.05-1.12)$ and bladder cancer rates $(\mathrm{HR}=1.08,1.03-1.14)$. For workers in excavating, grading, paving, and related occupations, a group expected to have high DEE exposure, positive lung cancer 
rates were observed overall $(\mathrm{HR}=1.37,1.25-1.49)$, among foremen/forewomen $(\mathrm{HR}=1.35,1.04-1.77)$, excavating and grading occupations $(\mathrm{HR}=1.37,1.18-1.58)$, labourers $(\mathrm{HR}=1.55,1.29-1.86)$, and non-specified excavating/grading/ paving occupations $(\mathrm{HR}=1.35,1.15-1.59)$. Non-significant positive rates of bladder cancer were also observed overall $(\mathrm{HR}=1.08,0.93-1.26)$, among excavating and grading workers $(\mathrm{HR}=1.13,0.88-1.45)$, and non-specified excavating/graving/ paving occupations ( $\mathrm{HR}=1.29,1.00-1.68)$.

Conclusion These results identify construction groups with high cancer risk, including excavating and grading occupations, potentially due to DEE exposure, though co-exposure to other carcinogens (e.g. silica) is possible. Targeted prevention resources could reduce exposure and subsequently occupational cancer risk, but would benefit from more detailed DEE exposure information.

\section{$0-32$ THE VALUE OF PLAIN CHEST RADIOGRAPH AS A DIAGNOSTIC TOOL FOR TB RELATIVE TO GENEXPERT AMONG EX-GOLD MINERS IN LESOTHO}

'Botembetume Maboso, Rodney Ehrlich. 'University of Cape Town, South Africa

\subsection{6/OEM-2021-EPI.39}

Background The World Health Organisation and many national guidelines for TB management recommend treatment initiation in the presence of symptoms such as cough, weight loss, night sweats and or fever, and radiological changes suggestive of $\mathrm{TB}$ with or without bacteriological confirmation. However, none of the studies that investigated the value of plain chest radiograph (CXR) has been done in the Southern African ex-gold miner population. Given the characteristics of this population - a high prevalence of silicosis, past TB and recurrent $\mathrm{TB}$ and post-TB lung destruction -application of the above recommendation may lead to unnecessary TB treatment. Objectives To assess the performance of the screening CXR in the diagnosis of active TB disease among former gold miners from the South African mines using GeneXpert as the reference standard.

Methods We analysed the medical history information, CXR, and GeneXpert test results in a group of ex-miners examined between 2017 and 2018 at Mafeteng Occupational Health Service Centre (OHSC), Lesotho. We excluded those on TB treatment and those within twelve months of TB-treatment completion at the time of the visit. CXRs were read by a medical doctor with training and experience in the reading of pneumoconiosis and TB. A set of 300 of the CXRs were cross-read by two occupational medicine specialists with mining medicine experience, with $80-90 \%$ agreement with clinic readings.

Results We analysed information on 2572 subjects. The prevalence of active TB on GeneXpert was 3\%, with CXR sensitivity 0.97 , specificity 0.41 , positive predictive value 0.05 , and negative predictive value 0.99 .

Conclusion A CXR negative for TB is a valuable tool in ruling out active TB disease among ex-gold miners. However, its poor predictive value, given the high background prevalence of silicosis and previous TB in this population, makes bacteriological confirmation necessary before initiating $\mathrm{TB}$ treatment in ex-gold miners.

\section{$0-72$ HOW TO PREDICT OBSTRUCTIVE SLEEP APNOEA IN PROFESSIONAL DRIVERS?}

${ }^{1}$ Elisabeta Pletea, Nicole Majery, Camelia Trandafir. 'STM (Senvice de Santé au Travail Multisectoriel), Luxembourg, Belgium

\subsection{6/OEM-2021-EPI.40}

Objective Developing better tools to screen obstructive sleep apnea (OSA) in professional drivers is fundamental in occupational health services, given the serious consequences this condition can have on their safety.

Methods 1928 professional drivers were screened for OSA for three years using Berlin and Epworth questionnaires. $10.3 \%$ were suspected to be positive for OSA and send for polysomnography tests. We had a return of 39 data. We analysed the results with the MAP index because it has shown a high sensitivity and specificity in studies on professional drivers. The MAP index is easy to use and combines selfreported apnea symptoms (snoring and cessation of breathing) as well as objective data like age, sex, and body mass index (BMI). As the apnoea symptoms regarding snoring and observed apnoea are often not properly reported, we included the questions of the Berlin questionnaire about tiredness in order to improve the predictability and calculated a MAP2. To determine diagnostic test accuracy, we calculated both MAP indexes with apnea-hypopnea index (AHI) criteria already published in articles. Sensitivity, specificity, negative and positive predictive values (NPV, PPV) and area under curve (AUC) for receiver operating characteristic (ROC) were analysed. Predictive utility of both indexes was examined by characteristic variables, age $(<50$ or $\geq 50$ years) and BMI $\left(<30\right.$ or $\left.\geq 30 \mathrm{~kg} / \mathrm{m}^{2}\right)$.

Results Employing the MAP_index1 for OSA, the sensitivity obtained was $63.3 \%$ and specificity was $41.2 \%$. The PPV was $58.3 \%$ and NPV was $46.7 \%$. The AUC was 0.524 (95\% CI $0.339-0.709)$. The accuracy was higher in younger versus older drivers (AUC 0.701 versus 0.620). Sensitivity for Map index 2 was $59.1 \%$ and specificity was $52.9 \%$. The PPV was $61.9 \%$ and the NPV was $50.0 \%$. The AUC was $0.560(95 \%$ CI $0.376-0.744)$.

Conclusions The MAP 2 was had lower sensitivity but higher specificity. It cannot be considered as a better tool to predict obstructive sleep apnoea.

\section{0-254 TEMPORAL TREND AND SPATIAL PATTERN OF MORTALITY FOR MALIGNANT PLEURAL MESOTHELIOMA IN TAIWAN DURING 1975-2019}

${ }^{1}$ Jing-Yi Wu, Tsung-Hsueh Lu, Lukas Jyuhn-Hsiarn Lee. ${ }^{1}$ National Cheng Kung University, Taiwan

\subsection{6/OEM-2021-EPI.41}

Introduction Malignant pleural mesothelioma (MPM) is a rare but aggressive malignancy associated with asbestos exposure for more than $80 \%$ of cases. The average latency of MPM is twenty to forty years. The history of asbestos use in Taiwan showed the massive importation during 1960-1980, but was under control by legislation since 1989, and was totally banned by legislation since 2018 . 